Boletim Científico do Instituto Agronómico do Estado de Säo Paulo

Vol. 25

Campinas, junho de 1966

$N .{ }^{\circ} 3$

\title{
ESTIMATIVA DA DISTRIBUIÇÃO DA ENERGIA SOLAR NO ESTADO DE SÃO PAULO $\left({ }^{1}\right)$
}

Dr. Admar Cervellini e Dr. Enéas Salati, engenheiros-agrônomos, Cadeira de Fisica e Meteorologia, Escola Superior de Agricultura "Luiz de Queiroz", e Hernani Godoy, engenheiro-agrônomo, Seção de Climatologia Agrícola, Instituto Agronômico

\section{SINOPSE}

Os autores analisam a distribuição da energia solar no Estado de São Paulo, área limitada pelas latitudes de $20^{\circ} \mathrm{S}$ a $25^{\circ} \mathrm{S}$. É apresentada uma equação média para determinação da radiação na superficie, partindo de dados de insolação para as condições da referida área. Utilizou-se o método clássico da correlação entre dados de actinógrafo e heliógrafo.

\section{1 - INTRODUÇÃO}

A idéia da utilização dos dados de insolação para cálculos de radiação na superfície do globo, nasceu de Angström (1), seguindo-se a êle, entre outros pesquisadores, Kimball e Hand (4), Black e colaboradores (2) e Macris (5). As equações propostas tiveram em comum o processo de seu estabelecimento, isto é, cálculo de regressão linear, utilizando dados registrados por radiômetros e heliógrafos. A maior parte destas equações são indicadas para áreas bastante grandes, abrangendo variações de latitudes de algumas dezenas de graus.

Pelo presente trabalho é apresentada uma equação, estabelecida pelo método clássico de correlação entre dados de actinógrafo e heliógrafo de algumas cidades do Estado de São Paulo, sendo sua aplicabilidade restrita à área limitada pelas latitudes extremas do Estado, isto é, $20^{\circ} \mathrm{S}$ a $25^{\circ} \mathrm{S}$.

Embora o número de dados disponíveis no momento seja bastante reduzido e existente somente para poucas localidades, determinamos a equação como válida para o planalto paulista. A validez da equação não foi, porém, testada para cidades do litoral, cujo

(1) Trabalho apresentado à XVII Reunião da Sociedade Brasileira para o Progresso da Ciência, realizada em Belo Horizonte, Minas Gerais, de 4 a 11 de julho de 1965. Recebido para publicação a 7 de outubro de 1965. 
regime de insolação é bastante diverso, por não se dispor de registros de actinógrafos para essas cidades. Acredita-se, todavia, que, $a$ priori, aquela equação possa ser utilizada também para essas regiões, uma vez que para seu estabelecimento levou-se em consideração ùnicamente a nebulosidade.

\section{2 - CÁlCULO TEÓRICO DA RADIAÇÃO SOLAR}

A radiação solar, $Q_{0}$, que atinge a área horizontal unitária no tôpo da atmosfera no tempo $t$, foi estimada pela integração da expressão (Smithsonian Meteorological Tables - 1951) :

$$
\frac{\mathrm{d} \mathrm{Q}_{0}}{\mathrm{dt}}=\frac{\mathrm{J}_{0}}{\mathrm{R}^{2}}(\operatorname{sen} \delta \operatorname{sen} \varphi+\cos \delta \cos \varphi \cos h)
$$

onde,

$$
\begin{aligned}
\mathrm{J}_{0}= & 1,94 \text { cal } \mathrm{cm}^{-2} \min ^{-1} \text { (Constante Solar). } \\
\mathrm{R}= & \text { raio vetor da Terra (distância do centro da Terra ao } \\
& \text { centro do sol, expressa em termos do comprimento do } \\
& \text { semi-eixo maior da órbita terrestre). } \\
\delta= & \text { declinação do sol. } \\
\varphi= & \text { latitude do observador. } \\
\mathrm{h}= & \text { ângulo horário do sol. }
\end{aligned}
$$

A energia que chega, em média, durante um dia de determinado mês foi calculada integrando-se a equação acima, fazendo $h$ variar do nascer ao pôr do sol e tomando $\varphi, \delta$ e $R$ como constantes.

A equação final utilizada foi a seguinte:

$$
\begin{aligned}
\mathrm{I}_{\mathrm{o}}= & \frac{60 \mathrm{~J}_{0}}{\mathrm{R}^{2}} \operatorname{sen} \delta \operatorname{sen} \varphi\left(\mathrm{t}_{2}-\mathrm{t}_{1}\right)+ \\
& +\frac{24}{\pi} \cos \delta \cos \varphi \operatorname{sen} \frac{\pi}{12} \mathrm{t}_{\mathrm{m}}
\end{aligned}
$$

sendo, $\mathrm{e}$

$t_{1}$ e $t_{2}$ as horas de nascimento e ocaso do sol, respectivamente,

$$
t_{m}=\frac{t_{2}-t_{1}}{2}
$$

Considerando que o Estado de São Paulo abrange latitudes entre $20^{\circ} \mathrm{S}$ e $25^{\circ} \mathrm{S}$, foram calculados os valores de $\mathrm{Q}_{\text {. }}$ para a latitude 
média $22^{\circ} 30^{\prime} S$ para se obter uma estimativa do valor teórico diário mensal. Os dados obtidos encontram-se no quadro 1, pelos quais se podem verificar as variações de $Q_{0}$ entre aquelas latitudes.

QUADRo 1. - Radiação solar, por centímetro quadrado e por minuto, que atinge a superfície horizontal no tôpo da atmosfera no dia 15 de cada mês

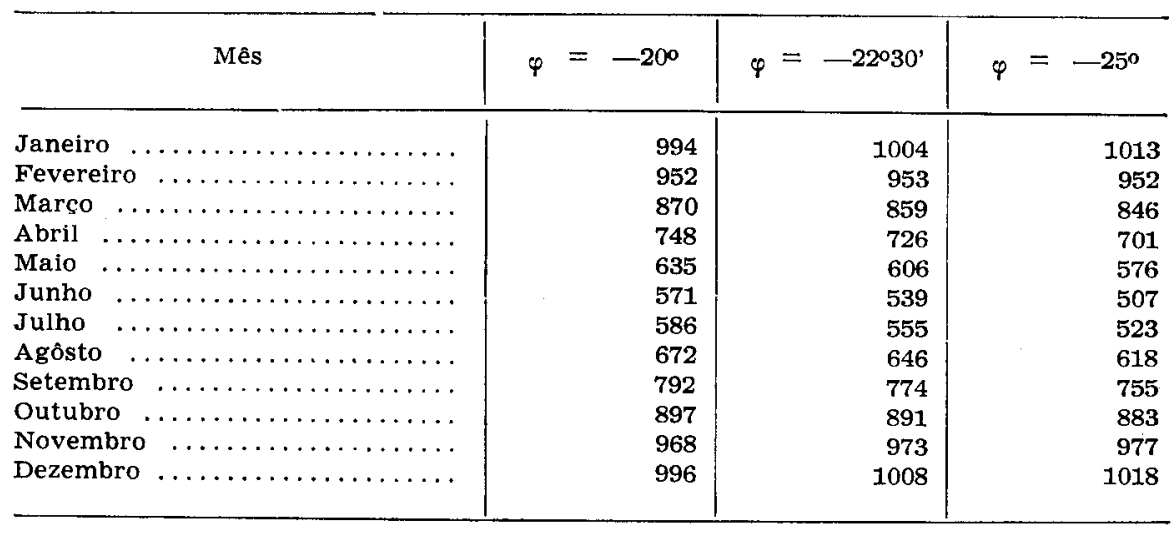

\section{3 - MATERIAL E MÉTODO}

Os dados utilizados para os cálculos de regressão foram fornecidos pela Seção de Climatologia Agrícola, do Instituto Agronômico de Campinas, sendo, os valores de insolação, obtidos com um heliógrafo, e os de radiação solar, com um actinógrafo Fuess.

Procurou-se estabelecer uma equação de regressão pelo método dos quadrados mínimos, que possibilitasse o cálculo da média diária da energia solar para cada mês (Q) em função da insolação $n$. O método geral é aquêle mesmo utilizado por Angström (1), Kimball e Hand (4), Black e colaboradores (2) e Macris (5).

Os dados foram analisados procurando-se estimar os valores de $a$ e $b$ da seguinte expressão:

sendo,

$$
\frac{\mathrm{Q}}{\mathrm{Q}_{\mathrm{o}}}=\mathrm{a}+\mathrm{b} \frac{\mathrm{n}}{\mathrm{N}}
$$

$\mathrm{Q}=$ radiação que chega, em média, num dia de determinado mês, por $\mathrm{cm}^{2}$, numa superfície horizontal na superfície do solo.

$\mathrm{Q}_{0}=$ radiação total do dia 15 de cada mês, tomado como representativo do mês, à latitude de $22^{\circ} 30^{\prime}$ no tôpo da atmosfera. 
$\mathrm{n}$ = insolação diária média mensal.

$\mathrm{N}=$ número de horas de insolação possível, para o dia 15 de cada mês, tomado como representativo do mês.

A análise da regressão foi feita pelo método dos quadrados mínimos, e a significância foi testada com um teste $F$ segundo Pimentel Gomes (3).

\section{4 - RESULTADOS E CONCLUSõES}

Foram obtidas, pelo método indicado, as seguintes equações de regressão :

Campinas (médias de 8 anos):

$$
\frac{\mathrm{Q}}{\mathrm{Q}_{\mathrm{o}}}=0,23+0,56 \frac{\mathrm{n}}{\mathrm{N}}
$$

Pindamonhangaba (médias de 9 anos) :

$$
\frac{\mathrm{Q}}{\mathrm{Q}_{\mathrm{o}}}=0,028+0,51 \frac{\mathrm{n}}{\mathrm{N}}
$$

Ribeirão Prêto (médias de 5 anos):

$$
\frac{\mathrm{Q}}{\mathrm{Q}_{\mathrm{o}}}=0,13+0,73 \frac{\mathrm{n}}{\mathrm{N}}
$$

Piracicaba (médias de 3 anos):

$$
\frac{\mathrm{Q}}{\mathrm{Q}_{0}}=0,18+0,62 \frac{\mathrm{n}}{\mathrm{N}}
$$

Monte Alegre do Sul (médias de 3 anos):

$$
\frac{\mathrm{Q}}{\mathrm{Q}_{0}}=0,19+0,61 \frac{\mathrm{n}}{\mathrm{N}}
$$

Mococa (médias de 2 anos):

$$
\frac{\mathrm{Q}}{\mathrm{Q}_{\mathrm{o}}}=0,40+0,41 \frac{\mathrm{N}}{\mathrm{n}}
$$




\begin{tabular}{|c|c|c|c|c|c|c|c|c|c|c|c|c|c|c|c|c|}
\hline$\stackrel{\mathcal{Q}}{\mathrm{a}}$ & 洛 & $\begin{array}{l}\text { 落 } \\
0\end{array}$ & 号 & 畧 & $\begin{array}{l}\text { 量 } \\
\text { 足 }\end{array}$ & $\begin{array}{c}\stackrel{3}{\sharp} \\
0\end{array}$ & $\begin{array}{l}0 \\
0 \\
0\end{array}$ & 몽 & 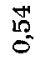 & 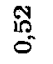 & $\begin{array}{l}\text { is } \\
0 \\
0\end{array}$ & $\begin{array}{l}\stackrel{H}{*} \\
0\end{array}$ & 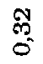 & $\underset{\sigma}{\mathscr{\sigma}}$ & 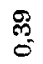 & $\frac{7}{0}$ \\
\hline 宫 & 点 & 5 & $\begin{array}{l}10 \\
\text { 点 } \\
0\end{array}$ & $\begin{array}{l}-1 \\
6 \\
0\end{array}$ & $\begin{array}{l}\text { I } \\
0 \\
0\end{array}$ & $\underset{\mathscr{Z}}{\mathscr{Z}}$ & $\begin{array}{l}8 \\
8 \\
0\end{array}$ & $\begin{array}{l}\infty \\
\stackrel{\infty}{0} \\
0\end{array}$ & $\begin{array}{l}\text { की } \\
\text { in } \\
0\end{array}$ & $\begin{array}{r}5 \\
0\end{array}$ & مo & $\stackrel{\mathscr{H}}{\stackrel{5}{6}}$ & के & $\begin{array}{l}\infty \\
\text { జ్ } \\
0\end{array}$ & 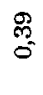 & $\stackrel{\infty}{\substack{0 \\
\sigma}}$ \\
\hline$\stackrel{+}{0}$ & $\underset{\substack{\infty \\
0}}{0}$ & $\begin{array}{l}10 \\
0 \\
0\end{array}$ & F & 品 & 占 & $\begin{array}{l}\text { m̊ } \\
0 \\
0\end{array}$ & $\stackrel{\mathscr{s}}{0}$ & 留 & $\begin{array}{l}8 \\
0 \\
0\end{array}$ & $\stackrel{\infty}{=}$ & $\underset{O}{\stackrel{g}{*}}$ & $\stackrel{\mathscr{H}}{\sharp 1}$ & 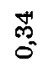 & $\begin{array}{l}\text { on } \\
\text { ó }\end{array}$ & సু & $\underset{\mathbb{N}}{\stackrel{\mathbb{S}}{0}}$ \\
\hline $\overrightarrow{\ddot{\nu}}$ & $\underset{8}{\stackrel{9}{0}}$ & 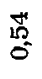 & $\stackrel{\text { 오 }}{\circ}$ & $\begin{array}{l}\infty \\
\substack{0 \\
0}\end{array}$ & ֻ̊ & $\begin{array}{l}\mathscr{e} \\
\text { ర్ } \\
0\end{array}$ & $\begin{array}{c}\mathscr{H} \\
\mathbb{H}^{\prime}\end{array}$ & $\stackrel{\overrightarrow{0}}{0}$ & $\frac{F}{\mathscr{L}}$ & 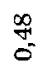 & $\begin{array}{l}\text { ํ⿱ 口⿱ 口心 } \\
0\end{array}$ & 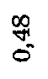 & 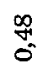 & : & $\underset{\text { N }}{\stackrel{N}{N}}$ & 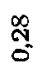 \\
\hline$\underset{8}{80}$ & ๙ั & $\stackrel{10}{8}$ & 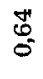 & $\frac{1}{5}$ & 5 & 告 & $\stackrel{8}{\circ}$ & $\frac{8}{5}$ & $\stackrel{\mathscr{B}}{\mathscr{B}}$ & $\underset{0}{E}$ & $\stackrel{8}{8}$ & 品 & 脜 & 吕 & 年 & 亏ั \\
\hline$\vec{B}$ & 둥 & ஜ̊ & $\underset{0}{0}$ & $\frac{\pi}{\tilde{0}}$ & $\begin{array}{l}+1 \\
0^{-1}\end{array}$ & $\begin{array}{l}\text { 瓷 } \\
\text { - }\end{array}$ & $\stackrel{8}{8}$ & F & $\stackrel{5}{6}$ & $\stackrel{\infty}{\stackrel{\infty}{\sigma}}$ & $\begin{array}{l}\infty \\
0 \\
0\end{array}$ & : & $\begin{array}{l}\text { 今 } \\
\text { O }\end{array}$ & 궁 & 是 & 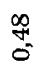 \\
\hline$\stackrel{S}{\stackrel{S}{S}}$ & $\stackrel{8}{0}$ & $\stackrel{8}{8}$ & $\begin{array}{l}\text { 용 } \\
0 \\
0\end{array}$ & $\frac{7}{5}$ & $\stackrel{s}{s}$ & 疍 & $\underset{\delta}{\mathscr{O}}$ & $\stackrel{0}{0}$ & $\underset{8}{8}$ & $\frac{8}{8}$ & $\stackrel{8}{8}$ & 8: & $\begin{array}{l}8 \\
0 \\
0 \\
0\end{array}$ & 今్ & $\begin{array}{l}\infty \\
\stackrel{\infty}{0} \\
0\end{array}$ & $\stackrel{\infty}{\mathscr{\sigma}}$ \\
\hline 丞 & $\begin{array}{l}\mathscr{8} \\
8 \\
8\end{array}$ & 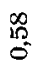 & $\begin{array}{l}10 \\
0 \\
0\end{array}$ & $\begin{array}{l}5 \\
0 \\
-\end{array}$ & $\frac{\mathbb{E}}{5}$ & 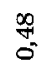 & $\begin{array}{l}10 \\
0 \\
0\end{array}$ & $\stackrel{8}{8}$ & $\begin{array}{l}8 \\
8 \\
0\end{array}$ & $\begin{array}{l}8 \\
8 \\
0\end{array}$ & 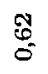 & 范 & $\begin{array}{c}6 \\
\text { 量 } \\
0\end{array}$ & $\underset{0}{\stackrel{Z}{\prime}}$ & $\stackrel{8}{\stackrel{1}{0}}$ & $\stackrel{\mathscr{2}}{\stackrel{+}{0}}$ \\
\hline$\frac{8}{4}$ & 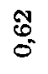 & $\begin{array}{l}\text { : } \\
\text { : } \\
0\end{array}$ & 楉 & $\begin{array}{l}8 \\
8 \\
8\end{array}$ & $\begin{array}{l}\mathscr{8} \\
\stackrel{8}{\circ}\end{array}$ & $\stackrel{5}{5}$ & : & $\begin{array}{l}8 \\
8 \\
0\end{array}$ & $\begin{array}{l}\infty \\
10 \\
0 \\
0\end{array}$ & $\mathscr{\delta}_{0}^{8}$ & $\begin{array}{l}8 \\
8 \\
0\end{array}$ & $\stackrel{\mathbb{R}}{0}$ & $\begin{array}{l}\text { 票 } \\
0\end{array}$ & $\stackrel{10}{\mathrm{~K}}$ & 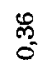 & 壳 \\
\hline 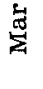 & $\begin{array}{l}10 \\
120 \\
0\end{array}$ & $\begin{array}{l}\text { : } \\
\text { : }\end{array}$ & 今ึ & $\begin{array}{l}\infty \\
10 \\
0 \\
0\end{array}$ & : & Iิ & $\begin{array}{l}10 \\
10 \\
0\end{array}$ & $\frac{8}{8}$ & 点 & $\begin{array}{l}10 \\
10 \\
0 \\
0\end{array}$ & 跑 & $\begin{array}{l}\stackrel{9}{\sharp} \\
0\end{array}$ & $\stackrel{\infty}{m}$ & \begin{tabular}{l}
0 \\
\multirow{6}{0}{}
\end{tabular} & ભొ & $\frac{19}{6}$ \\
\hline 离 & 与 & 广ே & 范 & 量 & 嗾 & 㤐 & 点 & $\stackrel{\mathscr{f}}{0}$ & ${ }_{0}^{10}$ & $\stackrel{5}{5}$ & $\frac{19}{7}$ & $\stackrel{7}{7}$ & $\begin{array}{l}\infty \\
\text { ๙ } \\
0\end{array}$ & 今్ & గ్రొ & $\begin{array}{l}\infty \\
0 \\
0 \\
0\end{array}$ \\
\hline 帤 & $\stackrel{51}{5}$ & $\begin{array}{l}0 \\
\text { 농 } \\
0\end{array}$ & 点 & 莺 & $\begin{array}{c}\text { i⿱ } \\
0 \\
0\end{array}$ & $\frac{\ddot{Z}}{0}$ & $\begin{array}{l}\text { Nิ } \\
\text { is } \\
0\end{array}$ & $\underset{\sigma}{5}$ & 总 & $\stackrel{\infty}{\stackrel{\infty}{0}}$ & 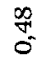 & 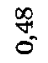 & के & $\begin{array}{l}\mathscr{H} \\
0\end{array}$ & 勇 & $\stackrel{\text { I }}{6}$ \\
\hline$\theta$ & 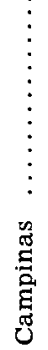 & 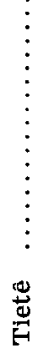 & 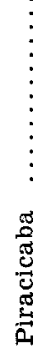 & 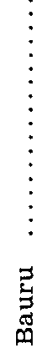 & $\underset{8}{\stackrel{\Xi}{8}}$ & 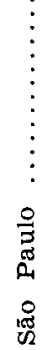 & 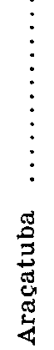 & 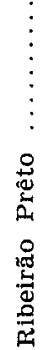 & 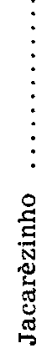 & 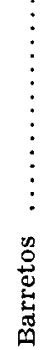 & 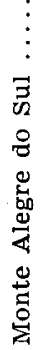 & 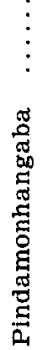 & 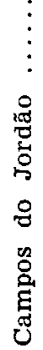 & 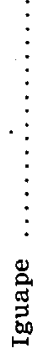 & 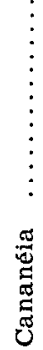 & 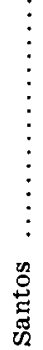 \\
\hline
\end{tabular}




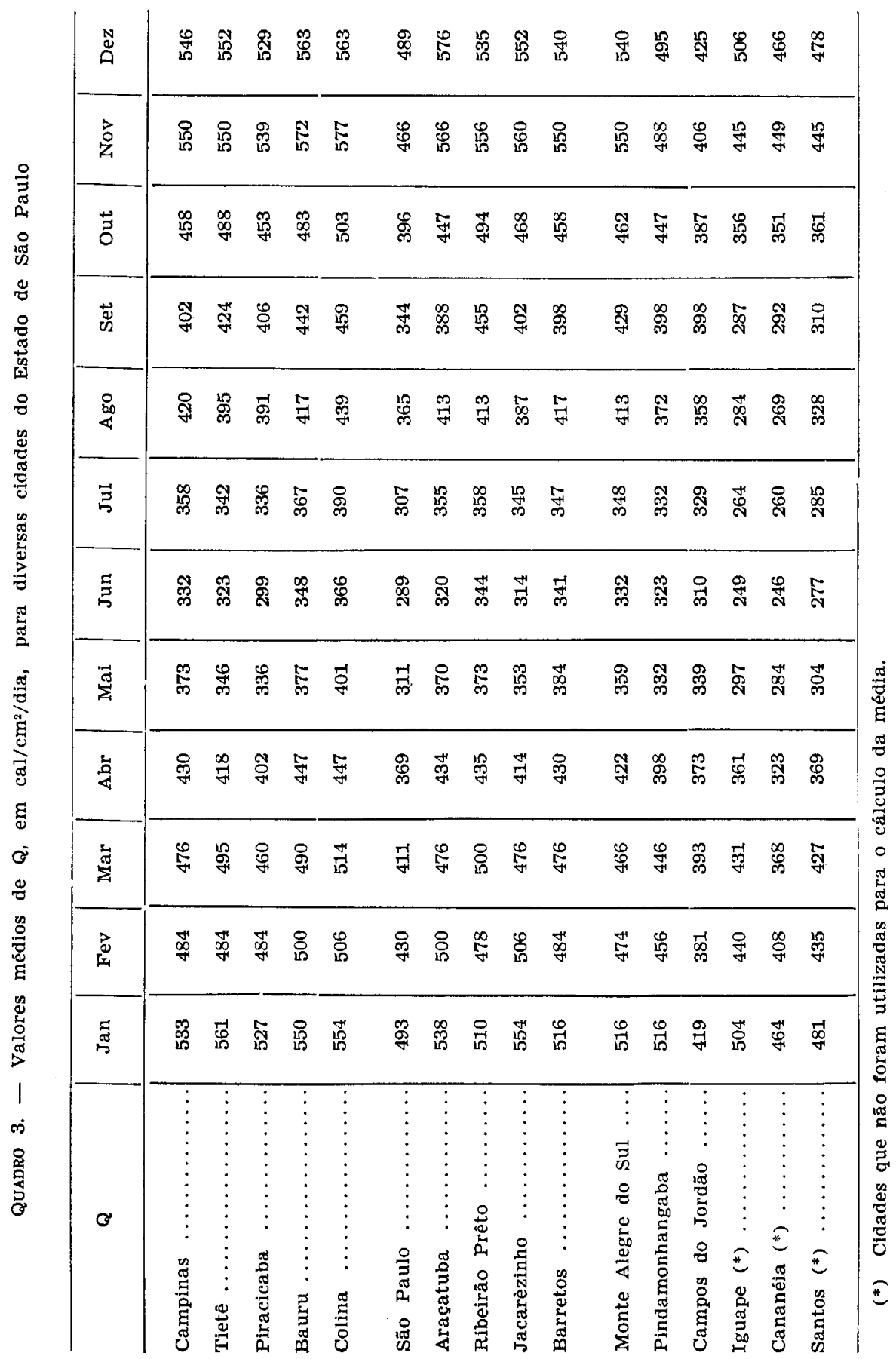




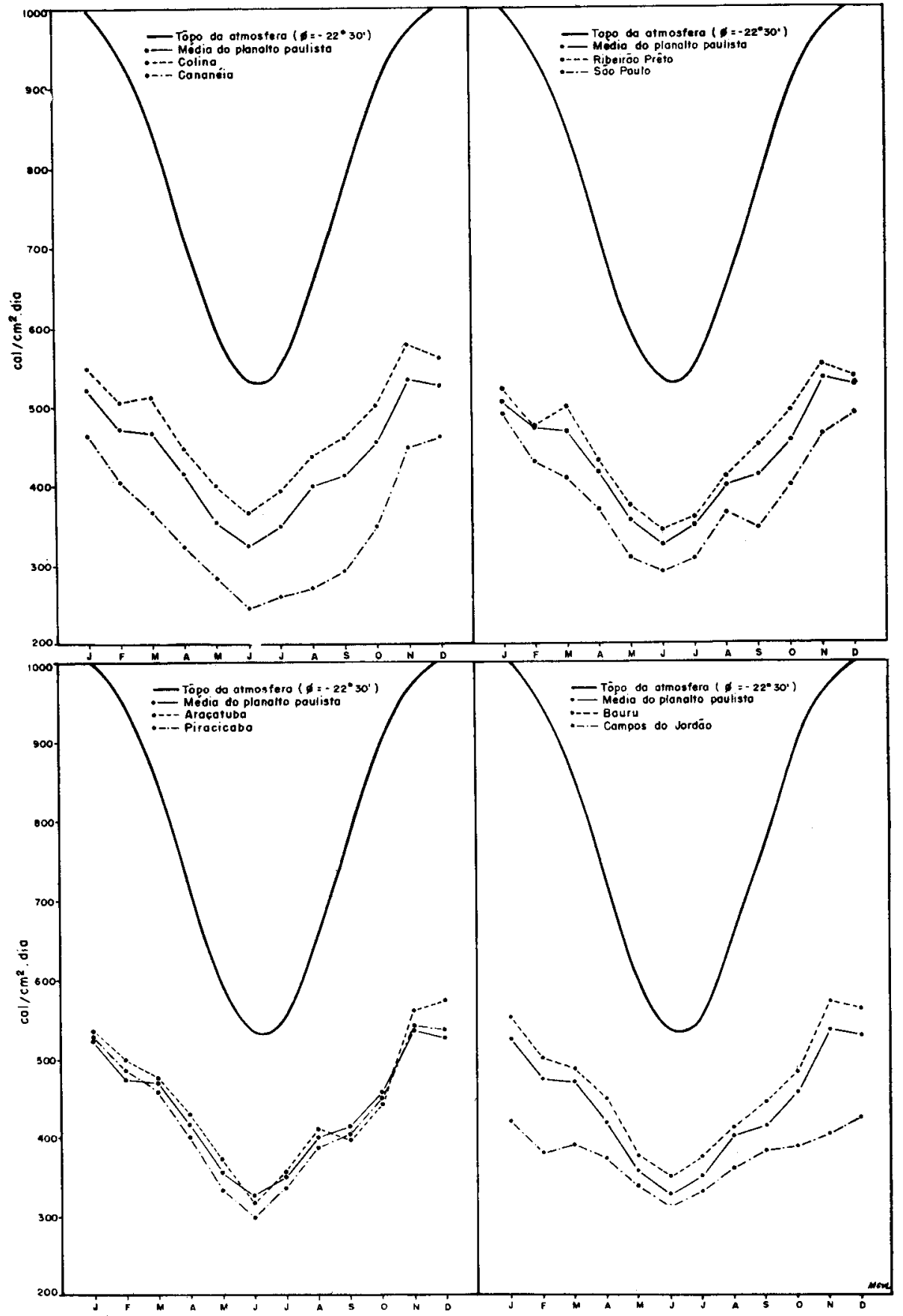

Figura 1. - Curvas anuais da radiação solar no tôpo da atmosfera à latitude de $22^{\circ} 30^{\prime} \mathrm{S}$ e calculadas a partir da insolação à superficie em diferentes localidades do planalto paulista. 


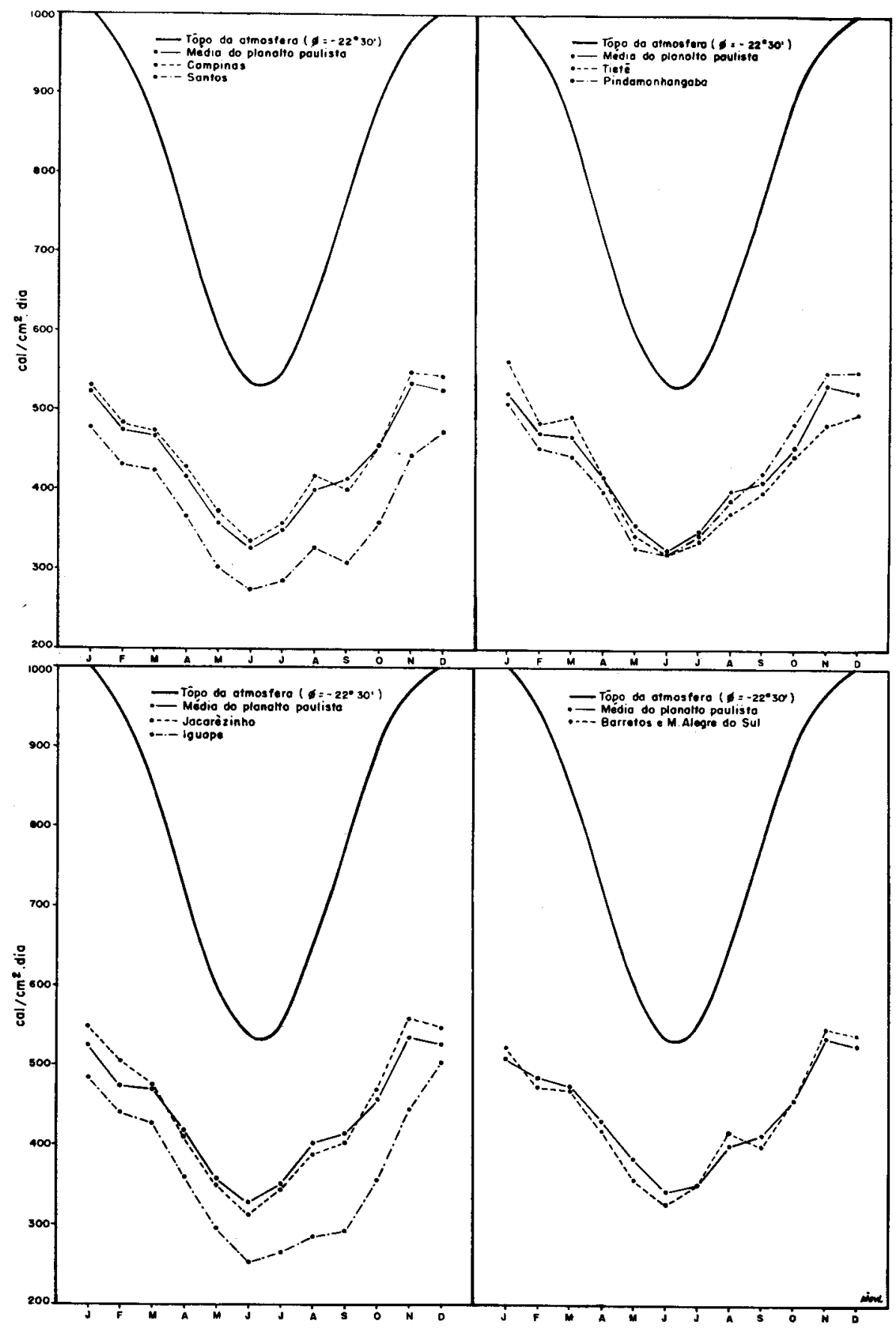

Figura 2. - Curvas anuais da radiação solar no tôpo da atmosfera à latitude de $22^{\circ} 30^{\prime}$ 's e calculadas a partir da insolação à superfície em diferentes localidades do planalto paulista. 
Procurando verificar se as equações acima poderiam ser reunidas numa só equação média, foram comparados, pelo teste de Tukey (3), os coeficientes $a$ e $b$ das equações de regressão para as cidades de Mococa e Ribeirão Prêto, que apresentavam maior discordância. Os valores obtidos, corrigindo-se os graus de liberdade segundo Sattertwate (6), demonstraram que as equações poderiam ser reunidas, obtendo-se, assim, a seguinte equação média para o Estado de São Paulo:

$$
\frac{\mathrm{Q}}{\mathrm{Q}_{\mathrm{o}}}=0,24+0,58 \frac{\mathrm{n}}{\mathrm{N}}
$$

Nas figuras 1 e 2 estão representados os valores para as diversas cidades comparados com a média.

Os dados obtidos de Q para as diversas cidades do Estado de São Paulo encontram-se no quadro 2, sendo calculados com os valores médios de 10 anos de $\mathrm{n} / \mathrm{N}$ das respectivas localidades (quadro 3 ).

\section{AN ESTIMATIVE ON THE DISTRIBUTION OF SOLAR ENERGY IN THE STATE OF SÃO PAULO}

\section{SUMMARY}

The authors analyse the distribution of solar energy in the State of São Paulo, an area which is limited within $20^{\circ}$ and $25^{\circ}$ south.

An average equation is presented for the determination of radiation on the surface, starting from insolation data for the conditions of the mentioned area. For this purpose the classic method was utilized of correlation between data from actinograph and heliograph.

\section{LITERATURA CITADA}

1. ANGStröm, A. Solar and terrestrial radiation. Journal of Royal Met. Soc. $50: 121-126.1924$.

2. Black, J. N., Bonython, C. W. \& Prescott, J. A. Solar radiation and the duration of sunshine. Quarterly J. Royal Met. Soc. 86(344): 231-235. 1954.

3. Gomes, F. Pimentel. Curso de Estatística Experimental. Publicação do Instituto de Genética da ESALQ-USP. 1954.

4. Kimball, H. H. \& HAND, I. V. Biological effects of radiation. New York, Ed. B. M. Duggar - McGraw-Hill Book Co.. 1936.

5. MacRIS, G. J. Solar energy and sunshine hours at Athens, Greece, Monthly Weather Review, 87:29-32. 1959.

6. Satterthwaite, F. E. An approximate distribution of estimates of variance components. Biom. Bull. $2: 110-114.1946$. 